\title{
Individual English Handwriting Synthesis
}

\author{
Riazat Ryan \\ Department of Computer Science \\ American International University - Bangladesh
}

\author{
Md. Haider Ali Kazal \\ Department of Computer Science \\ American International University - Bangladesh
}

\begin{abstract}
Handwriting is considered as an input method through pen-based or touch-based mean. Consequently, it is an unique feature preserving users' individuality. Since, it is becoming more lively aspect of user interaction, it is a very facile and more theoretical measure to reproduce an individual's cursive and noncursive English handwriting from ASCII transcription. Special input arrangement is designed to collect user's natural handwriting. Then, the system depicts the individuality features and characteristics of anyone's handwriting that machine learns afterwards. And at last, at a given set of instructions, for any set of ASCII value, user natural handwriting is synthesized hierarchically.
\end{abstract}

\section{General Terms}

Handwriting Reproduction, Human Computer Interaction

\section{Keywords}

Control point, Glyphs, Stroke, Ligature, Bezier Curve, 1-D Gabor Filter, Slant, Pressure, Semi Supervised Machine Learning

\section{INTRODUCTION}

With the advancement of technology, machine has become familiar with human handwriting as immediate inputs. Further, handwriting is regarded as a unique and authenticated 'signature' of an individual. So now, the emerged necessity for 'handwriting reproduction get-at-able technique' should be met up.

Several past works have been done very efficiently. Arabic handwriting generation [1], Bangla handwriting generation [3] have been done for other than English language.

This task is aimed only for English handwriting synthesis.

As for English handwriting regeneration, the motor model [9] showed handwriting process as a result of motor processes. Based on this motor model, another approach - delta-lognormal synergies [8] dealt with parametric definition in the context of kinetic theory. Subsequently, [13] modified delta-lognormal procedure with a conditional sampling algorithm for producing more smooth handwriting style. Along with this, Bayesian Network was used for handwritten character generation in [5]. An algebraic belta-elliptic model [2] has been conceived for understanding handwriting movement used in synthesis.
Handwriting is such a topic that a single feature can be considered for deepest exploration. For examples, [10] focused their work only on ligature modeling-one of the characteristics of cursive handwriting. Alongside, Cheng and Blumenstein concentrated on the improvement of segmentation of cursive handwriting [4].

As a summary to past works, this proposal is concise, naive, apprehensive and straightforward. With 3 consecutive steps-

\section{- Input arrangement \\ -Individuality feature extraction and learning \\ —Handwriting Synthesis}

Preserving a user's English handwriting style is the key point in this task. Sample handwriting are collected by the proposed pre-defined layout. For individuality features extraction, Bezier Curve, 1-D Gabor Filter have been applied. And, synthesis is done through a stroke generation method.

In addition, cursive, non-cursive both style can be regenerated in this case. All the figures resemble the only the visualization of the idea.

\section{INPUT ARRANGEMENT}

At very first, user's inputs are taken in a defined layout. Like in Figure 1, there will be a square box with both $\mathrm{X}$-axis and Y-axis inside it which will be used to take a single character input. That is- user, at the beginning, provides handwritten samples of each alphabet or character five times.

Since, all samples of each alphabet are provided individually, now on the second input arrangement step, the user has to imprint his cursive handwriting expression. For this, any random word is chosen from English dictionary and the system will ask the user to write down each character in a single box and thus the whole word in a sequence of boxes. For example, if there is 3 characters in a word, number of boxes will be 3 and they will look like the same as previous. In case of word inputs, the user has to go for 5 times as well.

If their style is non-cursive, same input arrangement is applicable.

A sample word 'act' is written in Figure 2 according to the layout. Input step ends here. Now, samples will be processed to extract specific properties. 

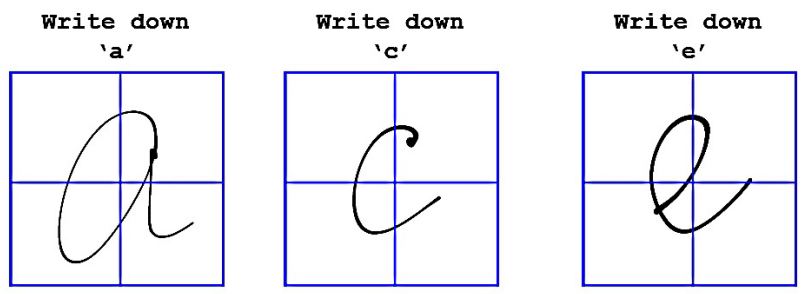

Write down

' $t$ '

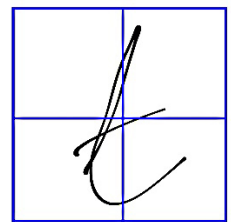

Fig. 1. Isolated handwritten alphabet samples

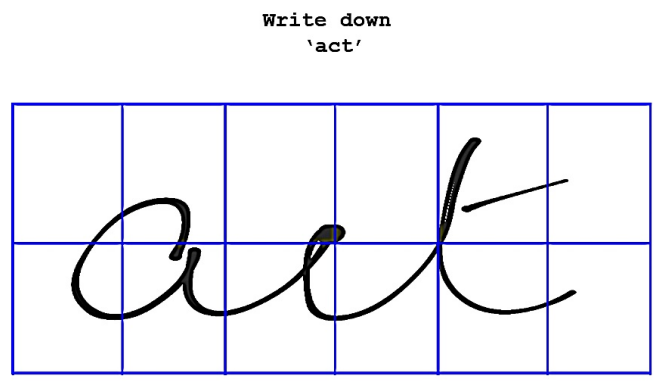

Fig. 2. Word - 'act' cursive handwritten sample

\section{INDIVIDUALITY FEATURE EXTRACTION AND LEARNING}

Individuality features of handwriting those can be computed with aid of machine and considered as unique for a user are character glyph, size, slant, stroke, ligature and pressure [6]. These 6 properties of handwriting have been considered for preserving the individuality of anyone's handwriting in this proposal.

Now, the proposed process of extracting individuality features and learning method are discussed below along with defining the aforementioned style preserving components.

(1) Slant: It is the position of a character strokes from the margin.

(2) Stroke: A pen-down to a pen up is a stroke. Below is an example of number ' 5 '. User used 2 strokes to write down ' 5 '.

(3) Size: The typical look of a character or present number of multiple strokes. We would discuss this within character glyph property.

(4) Character Glyph: It is a sequence of control points.

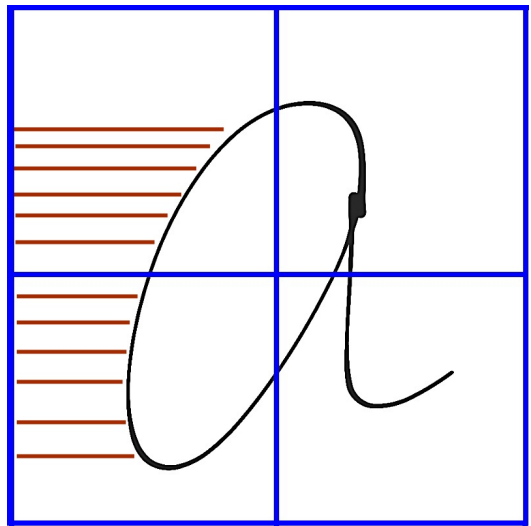

Fig. 3. Slant of user's handwritten letter 'a'

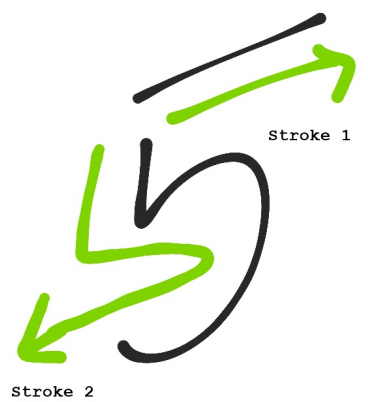

Fig. 4. ' 5 ' is written with 2 strokes

Control Points: Two end points of a stroke are control points. Each character glyph is consisted of multiple strokes and the eventually a glyph is nothing but list of control points on it.

So, to find out the elementary control points, Bezier spline curve comes first. Varga [12] used the Bezier curves and put those together in a series of Bezier arcs one after another over a character glyph in a pre-defined order. They manually put the basic control points like: the start and end point of the letter (1 in Figure 5), the points where tangent is horizontal or vertical ( 2 in Figure 5) and the corner points where the tangent is ambiguous ( 3 in Figure 5). Thus, key control points on a handwritten characters are fetched successively.

At this stage, having very few elementary control points, a few number of strokes of character 'a' can be generated. The more control points are there, the more strokes can be gained and thus a greater number of strokes can generate more natural looking user's handwriting. Cause, tiny little straight lines more in number can represent a perfect curve. So, the following step is to extract more control points using 1-D Gabor Filter.

Handwritten samples have X-component and Y-component. Moreover, it is a 1-D expression in real sense of the term. Wang [14] used the following equations of 1-D Gabor Filter on $\mathrm{X}$ and $\mathrm{Y}$ components and extracted more control points. This proposal went accordingly. 
Applied Bezier Curve on character ' $a$ '

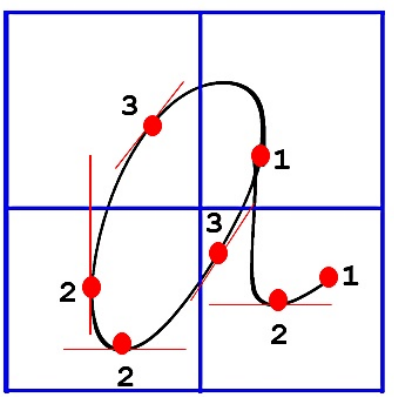

Fig. 5. Basic Control Points are extracted using Bezier Curve

$$
\begin{aligned}
& G \sin (x)=\text { const. } \exp \left\{-\frac{2 x^{2}}{T^{2}} \sin \left(\frac{2 \pi}{T} x\right)\right\} \\
& G \cos (x)=\text { const. } \exp \left\{-\frac{2 x^{2}}{T^{2}} \cos \left(\frac{2 \pi}{T} x\right)\right\}
\end{aligned}
$$

Here, $\mathrm{T}$ is the scale of the filter.

Thid 1-D filter takes out the strongest responsive control points. In addition, a series of multi-scale control points are extracted from handwritten glyphs as shown in Figure 6.



Fig. 6. 1-D Gabor Filter extracted more control points from character glyph 'a'

After control point extraction, there is now 5 samples of each character with all possible control points on it.

Words are actually sampled for ligature detection only. Ligature detection and learning process are discussed later on.
So, for each X-component of each control point we have 5 sample data since we have taken 5 sample inputs of each character from user. For, $X_{1}$, suppose the five samples are $X_{11}, X_{12}, X_{13}, X_{14}, X_{15}$.

Now, the Geometric Mean of $X_{1}$ is calculated.

$$
G \cdot M\left(X_{1}\right)=\sqrt[5]{X_{11} * X_{12} * X_{13} * X_{14} * X_{15}}
$$

The result is the new value for synthesizing $X_{1}$.

In Geometric Mean, items are multiplied. As a result, if, by any chance, variance between similar items gets greater, anomaly takes place. Similarly, if user handwritten character glyphs differs a lot in samples like- $X_{11}=2$ and $X_{12}=19$ then, the result will be an expected character glyph.

\section{Finding Geometric Mean of every $\mathrm{x}$ and $\mathrm{y}$ component of every node}

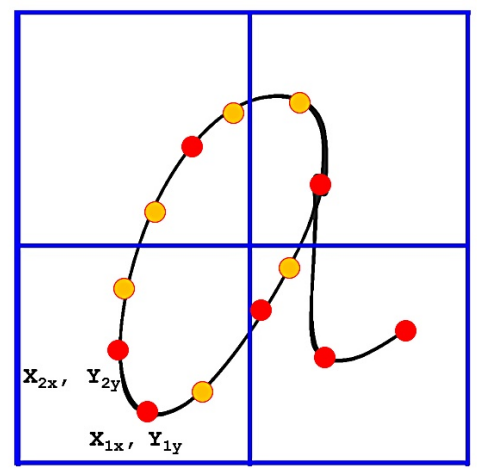

Fig. 7. Geometric Mean's X and Y components glimpse

Exact Geometric Mean process is applied to all $X_{2}, X_{3}, Y_{2}$, $Y_{3} \ldots$ sample components and the new $X_{2}, X_{3}, Y_{2}, Y_{3} \ldots$ will be the new control points of our synthesized character glyphs.

Control Points have been analyzed. So, now it can be estimated that how many strokes should be regenerated for synthesizing a particular character. Position of the points discloses the slant factor. Size feature has also been settled up because the proposal can comprehend about he number of control points needed to reproduce user's typical sized character glyph.

(5) Ligature: It is the part that connects a character with its' neighboring letter.

According to this concept, a ligature is a stroke between preceding character's end control point and succeeding character's first control point. Plus, there would be a direction of the ligature stroke and that will be the slope of that stroke.

On ligature detection, control points of ligature are processed exactly like other control points. The new angle value will be the average of 5 inputs' angle and from there the slope of ligature $\tan \theta$ would be traced. 
Ligature Detection and Learning

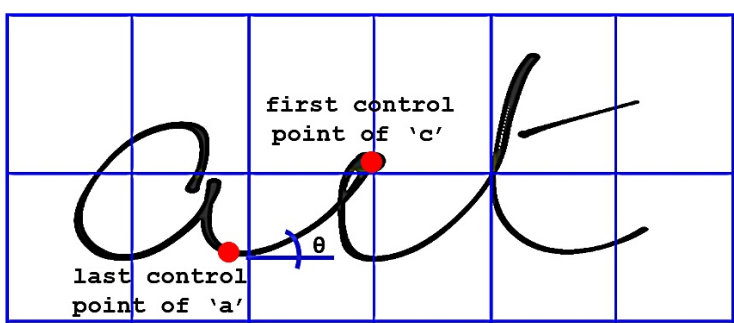

Fig. 8. Ligature Extraction

(6) Pressure: Measurement of how the user presses the pen against any paper or screen.

The pressure is calculated from sample inputs according to Number of black pixels[11] way. Yet, adjustment took place. For any input media such as - finger, touch pen or cursor there should be a threshold value of thickness of the drawn lines. If density of black pixel within a stroke is greater than the threshold value then pressure is fine. Otherwise, if the density is lower than the pre-set value then pressure is less than average.

Figure 9 describes the idea in detail.

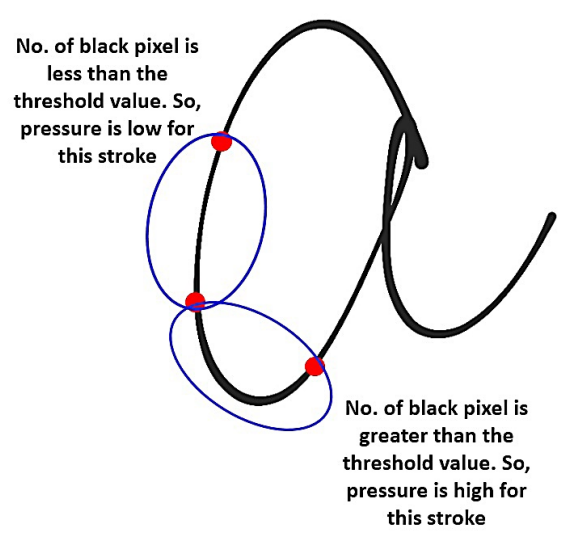

Fig. 9. Stroke's Pressure Explanation

Thus, for every stroke a pressure is assigned.

The feature extraction and learning process ends here. Now, it is to regenerate user's natural handwriting.

\section{HANDWRITING REGENERATION}

There are now the number of control points, the number of strokes and ligatures. Stroke has been considered as a unit in this proposal. A basic common method has been conceived to regenerate strokes as well as the ligature.
Stroke Regeneration: Position of the control points, i.e.; Xcomponent and Y-component are known to your system. From there, so the direction can be found by calculating the slope of these two points.

Suppose, two control points are $\left(X_{1}, Y_{1}\right)$ and $\left(X_{2}, Y_{2}\right)$. Slope of two points,

$$
S=\frac{Y_{1}-Y_{2}}{X_{1}-X_{2}}=\tan \theta
$$

So, two control points and their direction are there. The only thing needed is the curvature orientation - whether it is concave or convex.

Plamondon[7]'s Curve $\vec{\Sigma} \Lambda$ model presented a way to find out the curvature. According to the aforementioned model,

Let, $\theta_{i e}$ be the direction of $\left(X_{2}, Y_{2}\right)$ point and $\theta_{i s}$ be the direction of $\left(X_{1}, Y_{1}\right)$ point. Differences between their direction is the stroke line. Their magnitude vector has been superseded by slope factor in our proposal.

And the curvature becomes now,

$$
C=\frac{\theta_{i e}-\theta_{i s}}{S}
$$

If the slope is negative, resulting curvature states negative. For the state of the art, a negative curvature is a concave curve and a positive curvature is a convex curve.

Eventually, all through every tool-control points, strokes and curvature for regenerating English handwritten glyphs are ready hereby. And now, for each ASCII transcription, user's natural looking, style preserving handwritten glyphs will be reproduced hierarchically.

Ligature has also been considered as a single stroke. Their characteristics are the same like typical character strokes. So, ligatures can also be produced this way. If user practices non-cursive handwriting then ligature extraction as well as regeneration would be absent.

\section{CONCLUSION AND FUTURE WORK}

This is a user-centric work. This study was conducted for the purpose of regenerating user's English handwriting in an easy going way. Samples have been put in a array first. Style preserving properties are computed and learned with some specific tools then. At the end, a simple stroke generation method reproduced the individual English handwritten characters successively. Since, ligature and strokes are the same here, so both cursive and non-cursive handwriting can be synthesized with the same generation method.

Handwriting is, indeed, an interactive way in between a smart device and a user. More flowing techniques can make this input way much more fancier.

To discuss its' future, it can be said that, this work can be prolonged to generate any other language. Input techniques can be drawn up to more effortless step. Integration of more sophisticated tools can compute more individuality factors. As a result, synthetic handwriting would be more natural. Ligature detection and modeling - is the 
field to go far detail. If tilt of the input tool could be calculated then results would have been more noble. Finishing stroke generation algorithm could consider more parameters for synthesis.

\section{ACKNOWLEDGMENT}

Gratitude to our beloved thesis supervisor Dr. Khandaker Tabin Hasan, Associate Professor, Department of Computer Science, American International University -Bangladesh. His precious suggestions in this task led to accomplishment of this job in a more acceptable form. His patient with this work added boost to the daily efforts. No doubt, his generous supportive temperament favored all the way.

Also, Md. Rokon Uddin's potential HCI context support made the journey ceaseless.

\section{REFERENCES}

[1] Husni Al-Muhtaseb, Yousef Elarian, and Lahouari Ghouti. Arabic handwriting synthesis.

[2] Hala Bezine, Adel M Alimi, and Nasser Sherkat. Generation and analysis of handwriting script with the betaelliptic model. In Frontiers in Handwriting Recognition, 2004. IWFHR-9 2004. Ninth International Workshop on, pages 515-520. IEEE, 2004.

[3] Bidyut B Chaudhuri and Abhisek Kundu. Synthesis of individual handwriting in bangla script.

[4] Chun Ki Cheng and Michael Blumenstein. Improving the segmentation of cursive handwritten words using ligature detection and neural validation. In Proceedings of the 4th Asia Pacific international symposium on information technology (APIS 2005), Gold Coast, Australia, pages 56-59. Citeseer, 2005.

[5] Hyunil Choi, Sung-Jung Cho, and Jin H Kim. Generation of handwritten characters with bayesian network based on-line handwriting recognizers. In 2013 12th International Conference on Document Analysis and Recognition, volume 2, pages 995-995. IEEE Computer Society, 2003.

[6] Zhouchen Lin and Liang Wan. Style-preserving english handwriting synthesis. Pattern Recognition, 40(7):2097-2109, 2007.

[7] Réjean Plamondon and Moussa Djioua. A multi-level representation paradigm for handwriting stroke generation. Human movement science, 25(4):586-607, 2006.

[8] Réjean Plamondon and Wacef Guerfali. The generation of handwriting with delta-lognormal synergies. Biological $C y$ bernetics, 78(2):119-132, 1998.

[9] Rejean Plamondon and Frans J Maarse. An evaluation of motor models of handwriting. Systems, Man and Cybernetics, IEEE Transactions on, 19(5):1060-1072, 1989.

[10] Bong-Kee Sin and Jin H Kim. Ligature modeling for online cursive script recognition. Pattern Analysis and Machine Intelligence, IEEE Transactions on, 19(6):623-633, 1997.

[11] Sargur N Srihari, Sung-Hyuk Cha, Hina Arora, and Sangjik Lee. Individuality of handwriting. Journal of Forensic Sciences, 47(4):856-872, 2002.

[12] Tamás Varga, Daniel Kilchhofer, and Horst Bunke. Templatebased synthetic handwriting generation for the training of recognition systems. In Proceedings of the 12th Conference of the International Graphonomics Society, pages 206-211, 2005.
[13] Jue Wang, Chenyu Wu, Ying-Qing Xu, and Heung-Yeung Shum. Combining shape and physical modelsfor online cursive handwriting synthesis. International Journal of Document Analysis and Recognition (IJDAR), 7(4):219-227, 2005.

[14] Jue Wang, Chenyu Wu, Ying-Qing Xu, Heung-Yeung Shum, and Liang Ji. Learning-based cursive handwriting synthesis. In Frontiers in Handwriting Recognition, 2002. Proceedings. Eighth International Workshop on, pages 157-162. IEEE, 2002. 